\title{
A Qualitative Study on the Unmet Needs of Cancer Carers in Vietnam
}

Chris Jenkins ( $\nabla$ c.jenkins@qub.ac.uk)

Queen's University Belfast Centre for Public Health https://orcid.org/0000-0003-3514-3581

\section{Hien Thi Ho}

Hanoi University of Public Health

\section{Hoa Le Phuong Nghiem}

Vietnam National Cancer Hospital

\section{Gillian Prue}

Queen's University Belfast

\section{Lynne Lohfeld}

Queen's University Belfast

\section{Michael Donnelly}

Queen's University Belfast

\section{Minh Van Hoang}

Hanoi University of Public Health

\section{Olinda Santin}

Queen's University Belfast

\section{Research article}

Keywords: Cancer, carers, caregiving, Vietnam, LMICs, global health, qualitative, NCDs

Posted Date: April 2nd, 2020

DOI: https://doi.org/10.21203/rs.3.rs-18831/v1

License: (c) (1) This work is licensed under a Creative Commons Attribution 4.0 International License.

Read Full License 


\section{Abstract}

Background: Due to limited resources in hospitals and social and cultural norms regarding caregiving in Vietnam, carers provide a range of supportive functions for family members diagnosed with cancer. This paper provides empirical evidence on the self-identified unmet needs of carers of inpatients in national oncology hospitals in Vietnam.

Methods: An international, multidisciplinary research team conducted focus group and in-depth interviews with informal carers $(n=20)$ and health care providers $(n=22)$ and collaboratively analysed the results using thematic content analysis.

Results: Thematic content analysis highlighted four main areas of needs: (i) material requirements such as accessible and comfortable facilities, accommodation and finance; (ii) information needs about cancer, caring, and nutrition; (iii) emotional support; and (iv) training about how to provide care to their family members during (a) treatment and (b) recovery phases.

Conclusions: Carers provide invaluable support in supporting people with a cancer diagnosis, particularly given wider systemic challenges in delivering cancer services in Vietnam. Increasing visibility and formal support is likely to have both a positive impact upon the health and wellbeing of carers, as well as for cancer patients under their care.

\section{Background}

There are an estimated 165,000 new cancer diagnoses and 115,000 cancer-related deaths in Vietnam per year (1). The most common cancers associated with mortality across both sexes are liver (23.48\%), lung (19.14\%), stomach (13.92\%), and breast (5.64\%). Research on cancer service delivery in Vietnam is limited, but indicates that there are significant challenges across the health system in providing quality cancer services (2-6).

Given the challenges in cancer service delivery, many people diagnosed with cancer are supported by informal carers. Informal carers are often considered as "relatives, friends, and partners who have a significant relationship with and provide assistance (i.e., physical, emotional) to a patient with a lifethreatening, incurable illness" (7).

In Vietnam, as in many lower and middle income countries (LMICs), carers fulfil several roles, from providing informational and emotional support, to assisting with medical procedures and navigating hospital and medical administration. They face numerous challenges in fulfilling these roles, while also supporting and caring for themselves in the process (8). In part, this is because there are no embedded or widespread support services or networks for cancer carers in Vietnam.

As Vietnam undergoes an epidemiological transition from communicable diseases to non-communicable diseases, the nature and burden of caring is likely to change dramatically. It has been well documented 
that cancer creates unique and significant challenges for carers. Research has increasingly shown the impact that caregiving can have on carers' physical, emotional and mental health (9). Stress, loss of control, anxiety and depression are all commonly experienced by cancer caregivers (8). Previous studies have also demonstrated that poor health of the carer may correlate to poorer health for patients $(10,11)$.

Research on the needs of informal carers in Vietnam is limited. The few studies that exist report on the needs of Vietnamese carers looking after people with dementia and/or Alzheimer's disease (12), stroke (13), and HIV $(14,15)$. Other studies have been conducted with Vietnamese carers living in North America $(16,17)$. To date, there are no studies on the unmet needs of carers of hospitalized cancer patients in Vietnam.

This paper describes the range of self-reported and unmet needs experienced by carers, and introduces possible solutions and mechanisms to improve support. The paper focuses particularly on the unmet needs of caring within a hospital environment. Given the importance of carers within the health system in Vietnam, it is important to understand the unmet needs of carers both in terms of their own health, and their ability to provide effective care for patients.

\section{Methods}

\section{Study Design and Data Collection}

A three-stage approach was designed to inform the focus of the study, the development of data collection procedures, and to improve rigour and credibility through validating, verifying and triangulating data emergent from the main study.

\section{Figure 1: Study Design}

Key informant interviews and focus groups discussions (FGDs) (phase 1) were conducted with healthcare providers and carers to inform the iterative and collaborative development of an interview schedule (see supplementary information). FGDs and in-depth interviews were then conducted as part of a descriptive qualitative study with carers and healthcare providers in central, national-level oncology hospitals in Hanoi and Ho Chi Minh City (phase 2). Our preference was to conduct FGDs, but in-depth interviews were deemed a more appropriate method for some senior HCPs due to pressures on their time.

Following these first two phases, further focus groups and in-depth interviews were facilitated in which the findings of the studies were presented to carers and healthcare providers for the purposes of verifying, validating and deepening the analysis of the results (phase 3 ). Carers participating in this phase had not previously been involved in the study. HCPs consulted in this phase had been previously involved in either phases 1 or 2 . 
The semi-structured FGDs and in-depth individual interviews were led by an experienced Vietnamese qualitative researcher, conducted in Vietnamese, and lasted between 60-90 minutes. All FGDs and indepth interviews were partially translated simultaneously by a second Vietnamese researcher to enable non-Vietnamese members of the research team who were present during the FGDs and interviews to suggest relevant probes and additional questions with minimum interruption to the interviews.

\section{Setting, Recruitment and Study Participants}

Carers $(n=20)$ were recruited via convenience sampling within hospital settings in Hanoi K Hospital and the Ho Chi Minh Oncology Hospital. Healthcare providers $(n=22)$ in four departments of the two hospitals (radiology, surgery, chemotherapy and palliative care) were invited to participate in the study by administrative and medical contacts. Carers were invited to participate in FGDs by healthcare workers at each hospital, and were recruited from different departments in the hospitals (e.g. radiology, surgery, chemotherapy, palliative care). Inclusion criteria were that participants were currently caring for cancer inpatients at the hospitals and were over the age 18. Recruitment and data collection for phase 2 took place in November 2018. All interviews and FGDs took place in either Hanoi K Hospital or Ho Chi Minh Oncology Hospital. Study participants were recruited until preliminary analysis of the data indicated that saturation had been reached for all major identified themes $(18,19)$.

\section{Ethical Considerations}

All participants were fully informed about how their data and information would be used within this study, and were fully aware of their right to not participate. All participants were provided with an information sheet and the purposes of the study were described in-depth. Trained counsellors were available should participants have required support during interviews or FGDs, although this option was not utilised by any of the participants. All transcripts were anonymised on production. Data was stored in accordance with data management policies of both research institutions involved in the study. Ethical approval was granted from the ethical review board of the Hanoi University of Public Health.

\section{Data Analysis}

The 'voice' of carers are prioritised within the analysis and are complemented and triangulated $(20,21)$ with data from key informants and stakeholders such as doctors and other healthcare providers. These results were corroborated with data gathered through dissemination and verification interviews (phase 3 ). All interviews and FGDs were transcribed verbatim in Vietnamese and fully translated into English for analysis by the whole team.

Thematic content analysis was conducted (22) and an inductive codebook was collaboratively created based on the themes that emerged from initial independent readings. Results were discussed extensively in both face-to-face and online meetings to improve rigour and reliability. Vietnamese members of the research team travelled to the UK for 10 days to contribute in-person to the analysis of the data. This 
face-to-face time between members of the multidisciplinary and international team allowed greater discussion, triangulation of results and key themes, and improved rigour within the analysis of results.

\section{Results}

The sample of carers $(n=20)$ (Table 1.) was predominately female $(65 \%)$, aged between $29-72$ years old, with all carers with one exception being direct family members (e.g. parents, siblings, children and family by marriage). Cancer sites included breast, colorectal, oesophageal, stomach, and ovarian. HCPs $(n=22)$ represented a broad range of medical staff from departments of surgery, radiotherapy, palliation, nursing, nutrition and social work, and included staff in both senior and junior positions.

Carers gave their time between their caring responsibilities. With the exception of two carers within our key informant interviews (phase 1) and one in our main study (phase 2), all carers were informal and not paid. Each participant was provided with a small financial compensation for his or her time in contributing to the study.

Table 1

Study participants by phase and role

\begin{tabular}{|llll|}
\hline Study Participants & \multicolumn{2}{l|}{ Study Phase } & \\
\cline { 2 - 4 } & $\begin{array}{l}\text { 1: Study Design } \\
\text { and } \\
\text { Development }\end{array}$ & $\begin{array}{l}\text { 2: Descriptive } \\
\text { Qualitative } \\
\text { Study }\end{array}$ & $\begin{array}{l}\text { 3: Data } \\
\text { verification and } \\
\text { validation }\end{array}$ \\
\hline Carers & 12 & 20 & 11 \\
\hline Doctors & 10 & 11 & 12 \\
\hline $\begin{array}{l}\text { Other Healthcare Professionals (Nurses, } \\
\text { Nutritional Dept., Social Work Dept.) }\end{array}$ & 13 & 11 & 16 \\
\hline Total & 35 & 42 & 40 \\
\hline
\end{tabular}

Four key themes were identified from the data. Unmet needs for carers in Vietnam were described in terms of material requirements such as accessible and comfortable facilities, accommodation, and finance. Other reported needs related to information on cancer, caring, and nutrition; emotional support needs; and training on how to provide care during treatment and recovery. These needs were identified across all three participant groups with little variation and confirmed during phase 3 of the study in which the analysis was validated and deepened without new themes emerging.

\section{Material Needs}

Both carers (C) and health care providers (HCP) described the hospital/inpatient context as challenging and difficult. Carers and HCP noted the heavily overcrowded nature of centralised oncology hospitals, 
resulting in patients often sharing beds. Participants in our study acknowledged that carers contribute to this overcrowding. Carers are expected to remain bedside to support the needs of patients, yet are often asked to leave the ward during working hours. Many carers often reported feeling resentful at being asked to leave their loved one's bedside for fear the patient would be neglected if they were not there to provide support:

My wife has not been able to walk since the surgery. In the days after that, I had to help her to use the toilet and to feed her, and I was still asked to get out (of the ward). Sometimes I got mad. I said, "Now you don't have anyone to care for the patient, but you still throw us out? We do not want to lie here, but we have to. If we are not here, who's holding the bedpan?" Do the doctors and nurses ever take the bedpan to the patients? $(\mathrm{C} / \mathrm{HN})$

Many carers and HCP reported that both carers and patients must travel long distances to reach nationallevel oncology hospitals in Hanoi or Ho Chi Minh City. This results in difficulties for carers who must find affordable accommodation near the hospital for the duration of the patient's treatment. Carers described situations in which they had to share motel rooms with other carers or stay in the corridors and communal areas of the hospital when asked to leave the wards. Often carers slept next to or under the patient's beds when they were in the wards. Some carers staying in nearby motels described fearing for their safety; others noted the risk of having their possessions stolen if they slept in the hospital:

The first time I spent up to tens of million for renting a motel room out there. The first days, I didn't know how to find a cheap motel. I came to a place up there and it cost me 300,000 VND per day (13 USD). I was shocked. It cost me a million [dong] for three days, without counting food costs. (C/HN)

Family members are not allowed to stay in the ward. They can only stay in the ward when needed. Family members have to take a mat to sleep outside in the corridor, and each time we come here for 25-30 days. I am the main caretaker. I sleep like that. I have aches and pains but I have to suffer. I am tired. (C/HCMC)

Costs for cancer treatment and services in Vietnam have been well documented. Economic challenges relating to the aforementioned accommodation and travel costs were additionally described as adding to the already difficult economic pressures experienced for families dealing with a cancer diagnosis:

My mom gets car sick, so she could not go by bus. Each time she went to Hanoi we had to pay 4 million [dong] for a taxi and 2 million [dong] for the train. (C/HN)

I think that most of the cancer patients that come here are from the provinces [rural areas]. We face difficulties in [terms of] travelling time. And worse is the financial problems. The majority of cancer patients are [living] in remote provinces and live in difficult conditions. We eat charity rice. We eat charity porridge. $(\mathrm{C} / \mathrm{HN})$

\section{Informational Needs}


Many participants described having a number of informational needs. These are categorised in two broad categories: information carers required about and for the patient related to their diagnosis, expectations for treatment, and questions about nutrition and traditional medicine; and information they needed as carers to both support themselves and provide logistical information on things like accommodation and places to stay near the hospital:

Even though we are family members, our knowledge about the field of cancer is still very limited. Now I have to take care of nutrition and psychological health.. My experience of patient care is very limited. $(\mathrm{C} / \mathrm{HN})$

Information needs for the patient were normally prioritised by carers in the study. Carers wanted information on a wide range of factors, from the aetiology of cancer, to information on the 'best' hospitals and doctors, to information on probability of survival:

We needed to choose a place to get treatment. For cancer, there are many places, but how do we find the best and most suitable? That is very important because there are many hospitals that have the ability to treat - like here, they focus on cancer treatment. I had to look around so that I knew that here is the hospital specialized in cancer. Before I didn't know the difference between hospitals. (C/HCMC)

Carers often requested simple information such as the stage of the patient's diagnosis and what this meant. They often reported a lack of information and communication between healthcare providers and carers and patients. This was often attributed to the lack of time that HCPs had for in-depth conversations. This problem is potentially exacerbated by patients relying on internet sources for information about cancer, and not knowing which sources to trust and which sources were accurate:

Basically, I'm not a person in the field [of cancer] so I do not have the knowledge. But the information on the Internet is too wide, so the knowledge we learn from the Internet is difficult to apply for the care of our family patients - what to eat, what not to eat, is milk allowed or not? Our information is not good; you don't know what to select to hear. (C/HCMC)

Information from different sources is not correct, thus the readers have the wrong understanding. So, when the patient asks questions, the doctor will explain the information to the patient accurately. But the patient will not accept the explanations of the doctor because what the doctor has said is different from what they have heard or read. (HCP/HCMC)

Doctors and healthcare professionals also described the difficulties in reiterating information to multiple carers. They referenced efforts made to create solutions to communication problems, such as the creation of regular group meetings organised by the hospital to try and answer common questions from carers.

When one person comes, they take care the patient. The next person comes, they also take care of the patient. So as a doctor, we feel very upset - doctors, nursing staff, head nursing staff and nurse aides... So they give medicines to a different person [caring for the same patient] each day. Sometimes the family 
has eight children; sometimes they have low awareness. They even sometimes they don't hand over the medicine [to the patient], which is the most important [task they have to do]. (HCP/HN)

Nutrition appeared to be a central concern for many carers. Carers described a feeling of confusion or uncertainty relating to what they should be feeding the patient and when. Carers wanted to know what food they should provide (carers provide all meals to patients) to help maximise patients' treatment outcomes and recovery. Specific dietary information was requested for different types of cancer, different stages of diagnosis, and different stages of the treatment process (e.g. food before and after surgery, food during chemotherapy, etc.):

The doctor is not able to advise you carefully about nutrition; they are only consulted about the [patient's] drugs or the daily necessities. I had to search the Internet, but the information from internet is not the same. $(\mathrm{C} / \mathrm{HN})$

Information needs to support the carer focused around training and on providing practical information on how to navigate the hospital administration, how to find cheap and comfortable accommodation, and how to support their emotional and mental health. It was suggested that resources such as booklets and online information should be created and provided to carers when they arrive in the hospitals.

\section{Emotional and Support Needs}

Carers described a wide range of emotional and psychological needs and a lack of specialised services available to help them cope with the strain and emotional impact of caring. Carers described feelings of stress, shock (at the diagnosis), fatigue, grief, and sadness that made it difficult for carers to look after themselves. HCPs additionally observed similar emotions and strains, and described how patients sometimes worry that their carer will abandon them due to the stresses they experience in their role:

I went to the second floor to cry hourly because she (her mother) was diagnosed at the late stage. [But] my mother is so old she may die on the operating table. So even with such surgery she cannot live long, either. It is very miserable psychologically. (C/HN)

Usually in the late stages, the patient is afraid of being abandoned because many caregivers have been helping for a long time. They are tired, and could not afford to pay anymore and they will abandon the patients. Patients are really afraid of that. (HCP/HCMC)

Carers additionally reported stresses upon relationships between carers and the patient, and of the lack of support groups and communities to address this problem. Most support for carers came from their peers on the wards or in motels close to the hospitals rather than from formalised services. Spousal conflict and challenges in decision making were frequently described:

Patients often feel uncomfortable. Husbands and wives also fight and argue... so we need to calm them down ... Some couples in the ward fight and swear at each other. I told the wife to forgive the husband 
[referring to the patient] because he has a disease and is under pressure. He refused to take drugs, swore and asked for death. So [I told his wife], "You have to say nice words, persuade him. (C/HCMC)

\section{Training needs}

Carers indicated a need for training and preparation for being a carer, particularly around certain clinical procedures. This included information on providing how to care for their family member and how best to support them during their treatment and recovery. Due to the crowded nature of hospitals, carers described having to undertake roles in providing medical treatment, for example holding chemotherapy bags, holding IV fluids, and changing dressings on wounds. Carers reported needing specific training to fulfil these roles, particularly given that they would need to continue some of these roles when the patient is discharged:

By the end of the week, the doctors and nurses are busy. If I call [for one of them], it takes them a very long time to get here because they are busy taking care of many people. Sometimes I have to learn how to do it myself. Sometimes I even want to learn the way to get the veins myself. They are so busy that sometimes they make mistakes. (C/HN)

I have asked the doctor very carefully, so when the doctor came, I asked him what is the best way to stop bleeding and how to treat hemorrhage. The doctor told us some methods, so now it's stable. But the thing is when I get home, I don't know how to treat. I have to ask the doctor how to stop bleeding temporarily so that I can bring the patient here. $(\mathrm{C} / \mathrm{HCMC})$

Along with specific training, carers highlighted the need for clearer signposting to different services and sources of information in the hospital. While some supportive resources and services have been developed (such as information sessions, online groups, leaflets and posters), often carers did not know how to access these resources or where to learn about what information is available. HCPs recognized the value of having carers who were trained and prepared, and suggested that training could be provided to carers to better support them. Although paid caregiving is uncommon in Vietnam, some HCPs suggested this was a possible area for growth:

The training of caregivers is very essential. Actually, it is necessary to have a school which organizes nursing classes or class for carers. (HCP/HN)

\section{Discussion}

Cancer carers in Vietnam are experiencing multiple unmet needs. These unmet needs often affect carer's health and influence the extent to which they can adequately care for patients. Needs are material, informational, and emotional.

Caring and family are largely synonymous and inseparable in Vietnam. One study exploring the needs of Vietnamese caregivers in Canada described the obligation to care for family members as the norm, stating, "It's like eating, you just do it' (17). Our study found similar themes and highlighted the 
importance of thinking about the needs of carers both individually and in terms of supporting the care of patients.

The nature and burden of caring in Vietnam has likely shifted dramatically within a short period. For much of history, caring would have been in response to (mainly) infectious and communicable disease. Such disease required care for short intense periods. Cancer, and many non-communicable diseases, change this model. The number of cancer cases in Vietnam is steadily increasing $(4,23)$, however, how caregiving is conceptualised remains largely unchanged. Carers are still expected to be present and central in the care of their family member, and are expected to sacrifice time, work, and often their own health in the process.

As shown within this, and many other studies (24), cancer requires time-intensive care for prolonged periods in specialised and centralised oncology hospitals. Cancer remains poorly understood in Vietnam, and there is low awareness concerning what to expect within both treatment and care (5). Cancer treatments are invasive and often permanent. Cancer is also expensive to diagnose and treat $(25,26)$. All these factors make the experience of caring for someone with cancer in Vietnam (largely) new. New forms of support have yet to be developed despite this need, and despite the changing burden of care, and as a result carers are living with a high level of sustained and unmet needs. Patient support networks are becoming increasingly established for people diagnosed with cancer in Vietnam. Such clubs are reported by patients to have provided significant emotional support and solidarity as patients navigate difficult shared experiences. Such clubs have not yet been established for carers, and may represent an area for future studies and interventions.

Information resources (both online and physical) along with the creation of communal rooms for carers to stay in when they have to leave the wards, should be considered. Such pragmatic, low cost interventions have the potential to greatly reduce challenges experienced by carers, and should be explored within future interventions. Training courses for informal carers may support capacity-building for carers and may also provide a point of contact for face-to-face dissemination of information specific to caring. Intervention testing training for dementia carers have been previously trailed with success (27), and could be explored in relation to the specific needs of cancer carers.

As this study was conducted in hospital settings it is likely not reflective of specific or different needs that carers experience at home and in the community. In-depth research that also explores different needs for people caring in the community should be explored. Given difficulties in accessing oncology hospitals due to location and costs, research on needs to the community may access carers with the most pronounced challenges. This study was additionally not large enough to reliably access differences in needs of carers of different ages, gender, and socio-economic position. Unmet needs were described in universal terms by study participants, but further research may be useful in highlighting specific needs for different groups of carers.

\section{Conclusions}


This paper highlights the unmet needs of family carers looking after cancer patients in Vietnam according to carers and health care providers. Carers described challenges they face in terms of lacking accurate information, requiring emotional support, training and signposting of different services. Interventions to test the effectiveness, acceptability, cost-effectiveness and scalability of physical and online informational and supportive resources would be an invaluable next step in supporting carers manage both the health of patients as well as supporting their own health and wellbeing. Integration and increased visibility and recognition of carers within the health care system would likely benefit both carers and the people they care for.

\section{Abbreviations}

C

Carers

FGD

Focus group discussion

HCMC

Ho Chi Minh City

$\mathrm{HCP}$

Healthcare provider

$\mathrm{HN}$

Hanoi

LMIC

Low/Middle income country

\section{Declarations}

Ethics approval and consent to participate: This study received ethical clearance from Institutional Review Board of Hanoi University of Public Health in Vietnam. No. 018-442/DD-YTCC. All participants in the study provided written consent for participation. All participants gave their consent for publication, and were fully informed about how their information would be used.

Consent for publication: $\mathrm{n} / \mathrm{a}$

Availability of data and materials: The datasets used and/or analysed during the current study are available from the corresponding author on reasonable request

Competing interests: The authors declare that they have no conflict of interest.

Funding: Funded through Department for Economy (UK), GCRF (Global Challenges Research Fund) administered through Queen's University Belfast. Ref: DFEGCRF18-19/Santin. The funding body had no role or influence in the study design, implementation or analysis. 
Authors' contributions: $\mathrm{CJ}$ led the analysis and writing of this paper. HTH and NLPH led data collection in focus group discussions and semi-structured interviews. OS and CJ contributed to data collection through asking questions within FGDs and interviews. CJ, OS, HTH and NLPH led data analysis with contributions, advice and support from LL, GP, MD and HVM. All authors read and contributed to drafting of the paper. OS lead the conceptual development of the study, with support from the wider team.

Acknowledgements: We'd like to offer our gratitude to the carers ad healthcare providers who shared their experiences and recommendations with us. Thank you to the management at both Hanoi K Hospital and Ho Chi Minh Oncology hospital for all your support in facilitating this study.

\section{References}

1. GLOBOCAN. Population Factsheet - Vietnam. IARC; 2018.

2. Jenkins C, Ngan TT, Ngoc NB, Phuong TB, Lohfeld L, Donnelly M, et al. Strengthening breast cancer services in Vietnam: a mixed-methods study. Global Health Research and Policy. 2019 Jan;4(1):2.

3. Minh HV, Ha BTT, Chuong NC, Anh ND. Women's health and health care in Vietnam. Health Care for Women International. 2018;39(4):364-7.

4. Pham T, Bui L, Kim G, Hoang D, Tran T, Hoang M. Cancers in Vietnam-Burden and Control Efforts: A Narrative Scoping Review. Cancer Control. 2019 Jan 1;26(1):1073274819863802.

5. Thuan TV, Anh PT, Tu DV, Huong TT. Cancer Control in Vietnam. Where are we? Cancer Control. www.cancercontrolinfo; 2016.

6. Vu Duy K, Minh H, Kim Bao G, Ng N, Nguyen V, Thanh Tuan L, et al. Views by health professionals on the responsiveness of commune health stations regarding non-communicable diseases in urban Hanoi, Vietnam: a qualitative study. BMC Health Services Research. 2018;18.

7. Applebaum AJ, Breitbart W. Care for the cancer caregiver: a systematic review. Palliat Support Care. 2013 Jun;11(3):231-52.

8. Northouse LL, Katapodi MC, Schafenacker AM, Weiss D. The Impact of Caregiving on the Psychological Well-Being of Family Caregivers and Cancer Patients. Seminars in Oncology Nursing. 2012 Nov 1;28(4):236-45.

9. Santin O, Treanor C, Mills M, Donnelly M. The health status and health service needs of primary caregivers of cancer survivors: a mixed methods approach. European Journal of Cancer Care. 2014 May 1;23(3):333-9.

10. Segrin C, Badger TA. Psychological and physical distress are interdependent in breast cancer survivors and their partners. Psychology, Health \& Medicine. 2014 Nov 2;19(6):716-23.

11. Kim Y, Kashy DA, Wellisch DK, Spillers RL, Kaw CK, Smith TG. Quality of Life of Couples Dealing with Cancer: Dyadic and Individual Adjustment among Breast and Prostate Cancer Survivors and Their Spousal Caregivers. Annals of Behavioral Medicine. 2008 Mar 18;35(2):230-8. 
12. Nguyen TA, Nguyen $H$, Pham T, Nguyen TH, Hinton L. A cluster randomized controlled trial to test the feasibility and preliminary effectiveness of a family dementia caregiver intervention in Vietnam: The REACH VN study protocol. Medicine (Baltimore). 2018 Oct 19;97(42):e12553-e12553.

13. Hayashi Y, Hai HH, Tai NA. Assessment of the needs of caregivers of stroke patients at state-owned acute-care hospitals in southern Vietnam, 2011. Prev Chronic Dis. 2013 Aug 22;10:E139-E139.

14. Lundberg PC, Doan TTK, Dinh TTX, Oach NK, Le PH. Caregiving to persons living with HIV/AIDS: experiences of Vietnamese family members. Journal of Clinical Nursing. 2016 Mar 1;25(5-6):78898.

15. Tran CT, Pham TH, Tran KT, Nguyen TKC, Larsson M. Caretakers' barriers to pediatric antiretroviral therapy adherence in Vietnam - A qualitative and quantitative study. Applied Nursing Research. 2017 Jun 1;35:1-5.

16. Meyer OL, Nguyen KH, Dao TN, Vu P, Arean P, Hinton L. The Sociocultural Context of Caregiving Experiences for Vietnamese Dementia Family Caregivers. Asian Am J Psychol. 2015 Sep;6(3):26372.

17. Donovan R, Williams AM. Care-giving as a Canadian-Vietnamese tradition: "it"s like eating, you just do it'. Health Soc Care Community. 2015 Jan;23(1):79-87.

18. Saunders B, Sim J, Kingstone T, Baker S, Waterfield J, Bartlam B, et al. Saturation in qualitative research: exploring its conceptualization and operationalization. Qual Quant. 2018;52(4):1893-907.

19. Strauss A, Corbin J. Basics of Qualitative Research: Techniques and Procedures for Developing Grounded Theory. Thousand Oaks: Sage; 1998.

20. Carter N, Bryant-Lukosius D, DiCenso A, Blythe J, Neville A. The Use of Triangulation in Qualitative Research. Oncology Nursing Forum: Quality Measurement and Improvement. 2014;41(5).

21. Farmer T, Robinson K, Elliott SJ, Eyles J. Developing and Implementing a Triangulation Protocol for Qualitative Health Research. Qualitative Health Research. 2006;16(3):377-94.

22. Taylor B, Francis K. Qualitative Research in Health Sciences: Methodologies, Methods and Processes. Routledge; 2013.

23. Nguyen SM, Deppen S, Nguyen GH, Pham DX, Bui TD, Tran TV. Projecting Cancer Incidence for 2025 in the 2 Largest Populated Cities in Vietnam. Cancer Control. 2019 Jan 1;26(1):1073274819865274.

24. Jenkins C, Minh LN, Anh TT, Ngan TT, Tuan NT, Giang KB, et al. Breast cancer services in Vietnam: a scoping review. Global Health Action. 2018;11(1):1435344.

25. Minh H, Phuong Pham C, Mai Vu Q, Ngo T, Ha Tran D, Bui D, et al. Household Financial Burden and Poverty Impacts of Cancer Treatment in Vietnam. BioMed Research International. 2017;2017:1-8.

26. Lan NH, Laohasiriwong W, Stewart JF, Tung ND, Coyte PC. Cost of treatment for breast cancer in central Vietnam. Glob Health Action. 2013;6.

27. Sousa L, Sequeira C, Ferré-Grau C, Neves P, Lleixà-Fortuño M. Training programmes for family caregivers of people with dementia living at home: integrative review. Journal of Clinical Nursing. 2016 Oct 1;25(19-20):2757-67. 


\section{Figures}
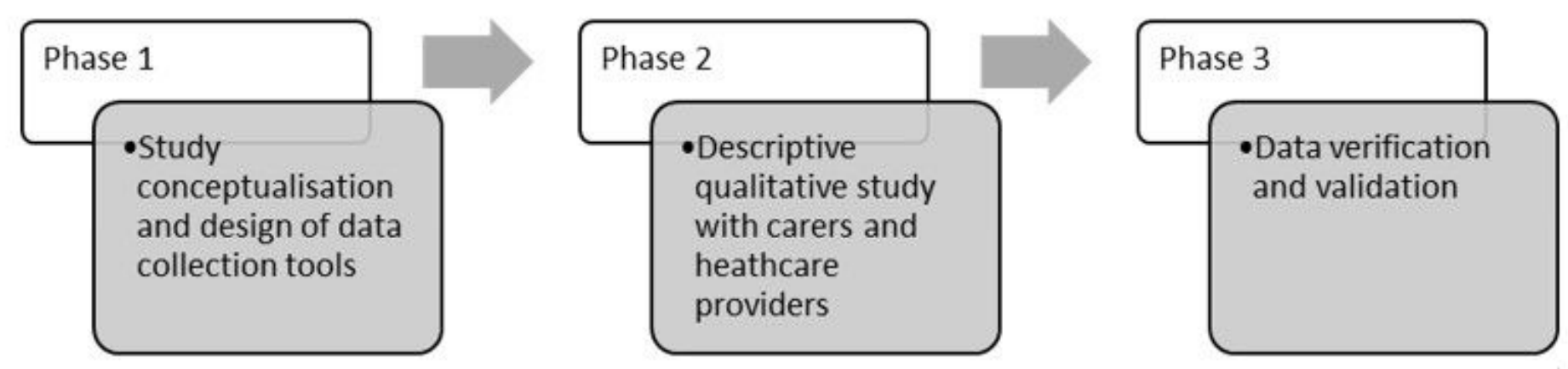

Figure 1

Study Design

\section{Supplementary Files}

This is a list of supplementary files associated with this preprint. Click to download.

- Supplementarylnformation.docx

- COREQChecklistCompleted.docx 